\title{
Safety Management of Civil Engineering Construction Based on Artificial Intelligence and Machine Vision Technology
}

\author{
Yuting Zhang $\mathbb{1}$ \\ School of Civil Engineering, Liaoning Technical University, Fuxin 123000, Liaoning, China \\ Correspondence should be addressed to Yuting Zhang; zhangyuting@st.btbu.edu.cn
}

Received 5 November 2021; Revised 24 November 2021; Accepted 30 November 2021; Published 13 December 2021

Academic Editor: Sang-Bing Tsai

Copyright (c) 2021 Yuting Zhang. This is an open access article distributed under the Creative Commons Attribution License, which permits unrestricted use, distribution, and reproduction in any medium, provided the original work is properly cited.

\begin{abstract}
With the development of social economy and the progress of science and technology, the process of urbanization continues to deepen and the progress of the construction industry changes with each passing day. People are paying more and more attention to the safety of civil engineering. Accidents frequently occurred during the construction process, which exposed the lack of attention to safety issues during the construction process, the lack of effective supervision of safe construction, and the lack of safety awareness of construction personnel, which had an impact on the construction of civil engineering. In this article, a solution to the safety management system for construction personnel based on artificial intelligence machine vision technology is proposed for the study of the safety management of civil engineering structures. First of all, through the research and analysis of the problems in the safety management of construction workers, the types of safety accidents of construction workers are summarized and the effect of machine understanding of the construction scene is realized through target detection and description of the spatial interaction between the two. A real-time detection and early warning platform is built, and early warnings are automatically sent out when a predefined dangerous scene occurs, so as to achieve the purpose of preventing construction accidents. The experimental results in this paper show that, in the use of artificial intelligence machine vision technology to manage the safety of civil engineering construction, the safety management of workers in the construction is realized, and the results show that the level of construction safety management has increased to $97.4 \%$, ensuring the quality of civil engineering construction and safety.
\end{abstract}

\section{Introduction}

According to the latest statistics from the State Administration of Work Safety, there were 773 construction accidents nationwide in 2019, and the death toll in the construction industry was as high as 904 , which are $5.31 \%$ and $7.62 \%$ higher than the numbers of accidents and deaths in 2018. In the UK, the number of workers in the construction industry accounts for only $5 \%$ of the total number of workers, but fatal accidents account for $27 \%$ of the total. In recent years, the government and industry have steadily increased their investment in management and construction safety, but there has been no significant improvement in methods and effects [1]. This situation is largely related to lack of consistency and insufficient basic research. Therefore, it is necessary to thoroughly study the law of accidents in the construction process, adopt new concepts and new technologies, and lay a solid foundation for adopting reasonable and effective measures to improve structural safety.

Improving the safety management level of workers in the construction industry will not only help construction companies better achieve the quality, time, cost, environment, and other management goals of construction management but also enhance the social enterprise image of construction companies and better survive and grow. However, current safety management still has problems, such as excessive reliance on employee observation, huge cost and time, insufficient samples to verify dangerous behaviors, and passive participation of employees. There are drawbacks. Therefore, it is very necessary to automate the monitoring or verification process. In recent years, more and more researchers have obtained information used to detect the behavior of machines and construction workers, control hazards, and avoid dangerous accidents through cameras 
installed on construction sites. Even if the currently used monitoring equipment and information management equipment can achieve a certain degree of automation and reduce human workload, these pieces of equipment can only be "visible" but cannot be "understood." The leap between the two often still requires the participation of engineering and technical personnel and requires the use of human knowledge and experience. The recent deep learning, especially the rapid development of image recognition technology, has made it possible for monitoring equipment to "understand." The artificial intelligence machine vision can manage more effectively and effortlessly, and relying on vision technology can better monitor safety accidents and ensure the safety of construction [2].

Machine vision is an excellent tool for inspecting various items such as textiles, fruits, printed circuit boards, electronic components, labels, integrated circuits, and machine tools. In the field of structural health monitoring (SHM), many innovative detection and monitoring systems based on machine vision technology have been developed. This technology has unique advantages such as noncontact, nondestructive, long-distance, high-precision, antielectromagnetic interference, and multipurpose monitoring. Ye et al. have proposed many machine vision-based structural dynamic measurement and structural state detection methods. Practical applications have also been developed to measure the physical parameters of the structure, such as displacement, tension/tension, and rotation, vibration, cracking, and shattering. The purpose is to summarize the basic theory and practical application of machine vision-based technology in structural monitoring, as well as the integration of system errors and other modern detection technologies. However, their research is not perfect in terms of error value and needs to be improved [3]. In the research work using this technology, Cubero et al. discovered the wavelengths that play an important role in detecting these hazards and thus developed a multispectral imaging system that can be used in industry. They reviewed recent work on the automatic detection of citrus using color and nonstandard computer vision systems and explained the different techniques available for acquiring images and their use in nondestructive testing. However, the method they proposed is still inadequate for image processing research [4]. Robie et al. described the various elements of machine vision analysis. A high-quality video recording method for automatic analysis, a video-based surveillance algorithm for evaluating interactive sites, and a machine learning method for detecting interactive patterns were proposed. However, the machine learning method that they proposed to recognize the interactive mode is still slightly insufficient in recognition accuracy [5]. In the context of development activities and increased public demand, construction activities have made major breakthroughs in the past two decades. Occupational health and safety issues continue to be the main concern of construction organizations. Due to poor safety management in the construction industry, the world society and economy have suffered economic and human losses. Benny and Jaishree discussed the main safety regulations and conducted a detailed study of the safety management procedures for construction sites. They also analyzed the different types of accidents that occurred on the construction site and the measures to control these accidents through the collection of various site visits, literature review, and various building safety standards data, including BIS and OSHA (Occupational Safety and Health Administration). However, their research lacks the sum of safety management accidents, and further research is needed [6]. During the implementation of these projects, due to unsafe road construction management techniques, many road workers, road users, and environmental problems have been caused. Nkurunziza targets three ongoing road construction projects in Kigali, namely, the Luliba-Kalama-Namiambo road, the Kigali urban road upgrade project in Agatare, and Sonatube-Ga on the Hanga-Akageira Highway; the existing problems were investigated to investigate the road construction safety management technology. From the survey results, $64.28 \%$ of the results indicate that the health and safety maintenance policies of road construction sites are applied, and there are different safety maintenance measures, of which $57.14 \%$ of project managers will provide workers with PPE (Personal Protective Equipment). Many workers did not wear them as required and found that $50 \%$ of accidents occurred during excavation. But the research results he got are still a bit one-sided, and he did not analyze them from all aspects of engineering [7]. Safety is the key goal of construction management, but construction safety management is complicated by the diversity of technology and management factors. If the key factors can be identified and corresponding measures can be taken, the improvement of safety performance will be more direct and effective. Zhang et al. used a systematic thinking method to treat construction safety management as a system, decomposing it into six subsystems and related management factors. The fuzzy fault tree analysis method was used to establish a reliability analysis model to reveal the failure probability of various factors in the safety organization management subsystem. Through a questionnaire survey conducted in Wuhan, China, they calculated the critical importance and average probability of basic factors. On this basis, nine key factors of the safety organization management subsystem were determined, and corresponding improvement measures were proposed, verifying that the construction safety management framework based on system thinking can be used as an effective tool to identify the causes of construction safety management failures. However, the safety issues included in the construction safety management system they studied are not comprehensive enough [8].

The innovation of this article is that it is based on artificial intelligence machine vision technology, improved feature extraction and image processing methods, and analyzed and compared the advantages and disadvantages of various algorithms; even in the case of blur, it can guarantee a better recognition rate, as well as enhancing the perception of rotation, zooming, noise, and other environments, selecting local feature extraction methods for safety awareness in urban engineering, and achieving rapid and efficient extraction of features in engineering construction. The safety issues and factors in civil engineering construction are analyzed, and the 
main factors and comprehensive factors are summarized to realize the safety management of construction [9].

\section{Method Design of Civil Engineering Construction Safety Management Based on Artificial Intelligence Machine Vision Technology}

2.1. Artificial Intelligence Machine Vision Technology. Machine vision is a rapidly developing field of artificial intelligence. It uses a machine to replace the human eye for measurement and judgment. The captured target is converted into an image signal through machine vision products [10-12], which is sent to a dedicated image processing system to receive the morphological information of the captured target and it is based on the pixel. The distribution digitally converts the signal into brightness, color, and other information, and the imaging system performs various functions on these signals to extract the characteristics of the target [13-15].

As an emerging research field, machine vision technology has developed rapidly, and research results have emerged one after another [16]. At present, the integration with industrial production is also the focus of manufacturing research [17]. In daily production, many fields are combined with machine vision technology. After continuous research and development, the characteristics of machine vision technology have been formed in various fields $[18,19]$. The system framework of artificial intelligence machine vision is shown in Figure 1.

As a kind of judging behavior, detection is based on the detection target, the detection standard is established, the detection object is compared with the detection standard, and the comparison conclusion is made. It can be seen from the above definition that testing is a cognitive process that judges and compares the value or pros and cons of an object with reference to certain standards.

2.1.1. Feature Extraction. Feature extraction is to convert the original features into a set of features with obvious physical meaning (Gabor, geometric features (corner, invariant), texture (LBP HOG)) or statistical significance or kernel and then find the most effective from the original features (invariance of samples of the same kind, discrimination of different samples, robustness to noise). Feature extraction belongs to the stage of image analysis and is the content of image editing [11]. Its function is to detect points of interest, straight lines, and other features in the image after the image is preprocessed and to create a description of the main parts of the image for subsequent understanding of the image $[20,21]$. The most typical function descriptors are floatingpoint vector descriptors and binary descriptors. The computational cost of binary descriptors is lower than that of floating-point descriptors, but they do not contain as much information as the first descriptor [22-24]. In addition, in complex environments such as industrial spaces, the image mismatch rate is high. The selection process of feature key points is shown in Figure 2.
The common features of the input image and the template are extracted and interpreted by a specific method, and a specific search strategy is used to crossspecify the matching primitives. Finally, the similarity measurement feature is used to determine the correspondence between the two images to complete the final recognition. Image attributes are consistent descriptions of image attributes. Compared with the amount of redundant data in the entire image, it reduces the difficulty and computational complexity of feature recognition in the application. Under normal conditions, it is stable to grayscale changes and blocking.

In the real world, there are certain obstacles in the acquisition of visual images in engineering, which brings about great inconvenience to the recognition process of the ground system. The image will be discarded. At this time, the local attributes need to be exported, and the two-dimensional Gaussian function is set as

$$
T(a, b, c)=\frac{1}{2 c^{2}} e^{a^{2}+b^{2} / 2 c^{2}} .
$$

After Laplace transformation is performed,

$$
\begin{aligned}
\delta^{2} t & =\frac{\phi^{2} t}{\phi a^{2}}+\frac{\phi^{2} t}{\phi b^{2}}, \\
\delta_{\text {norm }}^{2} t & =\phi^{2}\left(\frac{\phi^{2} t}{\phi a^{2}}+\frac{\phi^{2} t}{\phi b^{2}}\right), \\
& =-\frac{1}{a \phi^{2}}\left(1-\frac{a^{2}+b^{2}}{2\left(\phi^{2}\right)}\right) e .
\end{aligned}
$$

After transforming the function $T(a, b, c)$, you can set the value and extract the relevant information.

When $\phi^{2}$ is smaller, the number of focus points obtained increases significantly, and noise points will be mixed into the detection result. When the local small window is translated, its change is

$$
s(a, b)=\sum_{a, b \in W(a, b)}^{w} \phi^{2}(a, b) .
$$

Here, $\phi^{2}(a, b)$ is the Gaussian weighting function, and formula (3) can be expanded to obtain

$$
\begin{aligned}
& s(a, b)=\left[a^{\prime \prime}, b^{\prime \prime \prime}\right] t(a, b)\left[\begin{array}{l}
\nabla a \\
\nabla b
\end{array}\right], \\
& S(a, b)=\sum_{a, b \in w(a, b)} t(a, b)\left[m_{x}(a+b)^{2}\right] .
\end{aligned}
$$

The algorithm's judgment on strong features can be expressed as

$$
S_{\min }\left(\sigma_{1}, \sigma_{2}\right)>\sigma,
$$

where $\sigma_{1}, \sigma_{2}$ is the value in the autocorrelation table which is considered to be the defined threshold; it has local matching information and has small memory requirements. 


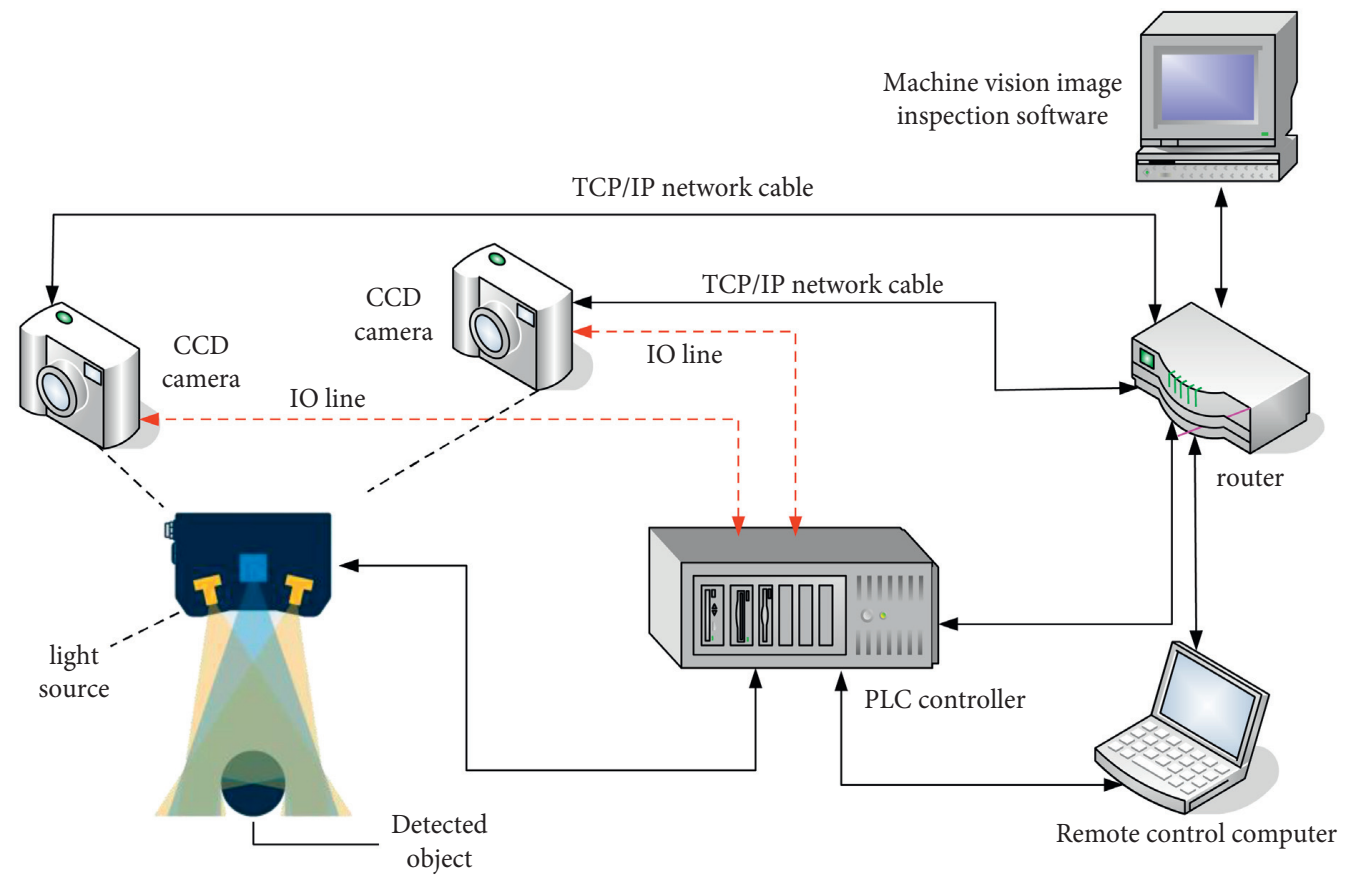

FIgURE 1: Machine vision system block diagram.
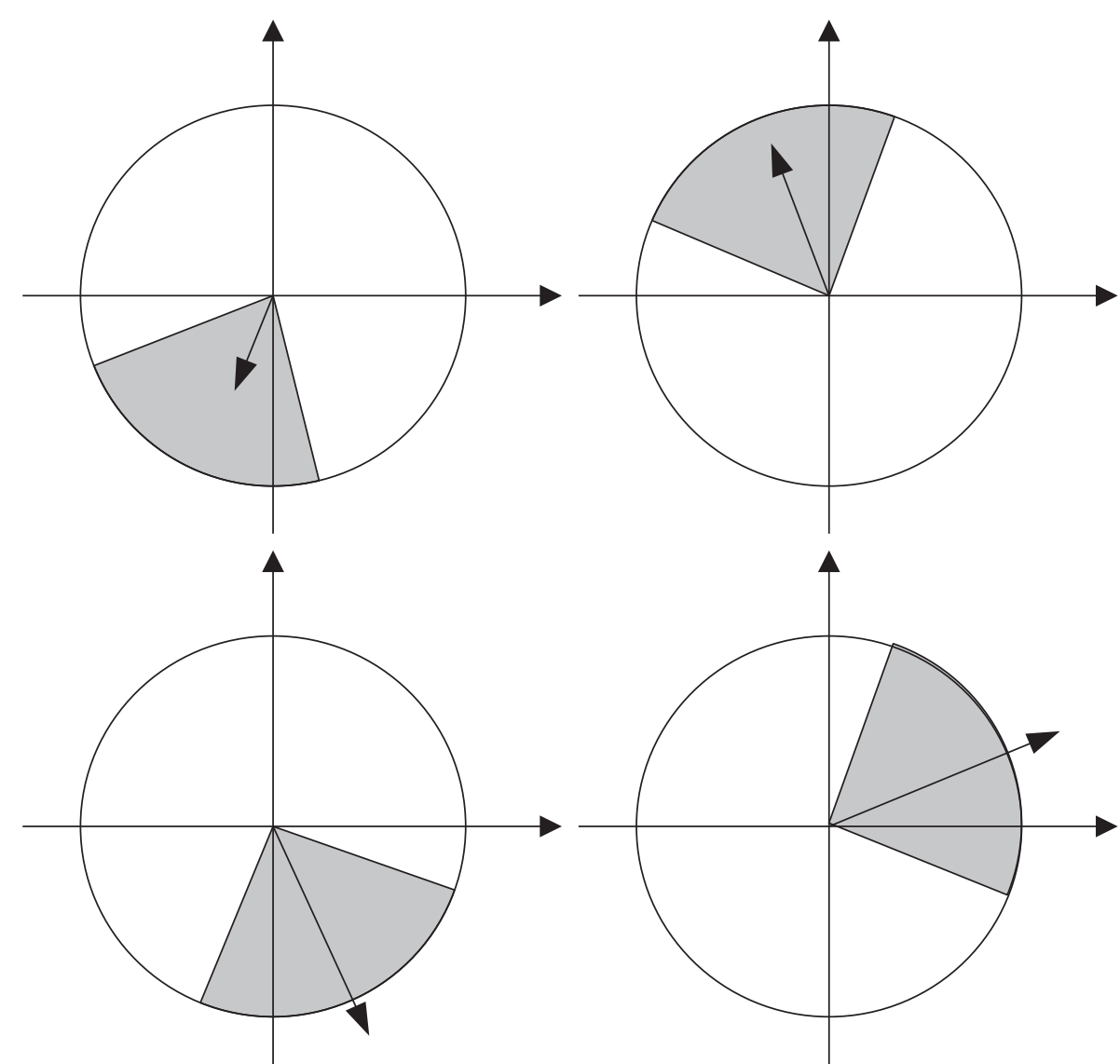

Figure 2: Selection of feature point direction. 
2.2. Image Preprocessing. Due to the imperfections of imaging systems, transmission media, and recording equipment, digital images are often contaminated by various noises during their formation, transmission, and recording. Generally, the noise signal is not related to the object to be studied; it appears in the form of useless information, disturbing the observable information of the image. Processing the image can effectively remove the noise in the target and background; at the same time, it can well protect the shape, size, and specific geometric and topological structure characteristics of the image target and extract the image characteristics for image recognition.

2.2.1. Parameterized Evaluation Method. This article introduces some commonly used parameterized indicators for quantitative evaluation of processed images of workpieces. Let $h(a, b)$ be the image of size $X * Y$, and let $g(a, b)$ be the processed image. For pixels $(s, t)$ in the image, the commonly used evaluation parameters are as follows.

Mean square error is as follows:

$$
M=\frac{\sum_{s=1}^{x} \sum_{t=1}^{y}[h(s, t)-g(s, t)]^{2}}{X \times Y} .
$$

The smaller the value of MSE, the better the image quality.

$$
N=10 \times \lg \frac{\left(h_{\max }-h_{\min }\right)^{2}}{M} .
$$

Here, $h_{\max }$ represents the maximum gray level and $h_{\min }$ represents the minimum gray level.

The larger the value of $N$, the better the effect of image processing. In order to improve the performance of the algorithm in real time and reduce the difficulty of processing, this article grays out the RGB image. The specific methods are as follows:

$$
h(s, t)=R(s, t) G(s, t) B(s, t) .
$$

Use the average value of the RGB elements of a specific pixel as the gray value of the point; then,

$$
h(s, t)=\max R(s, t) G(s, t) B(s, t) .
$$

Calculate the average value of the RGB elements of the color image with different weights as the gray value of the point:

$$
h(s, t)=z_{1} R(s, t)+z_{2} G(s, t)+z_{3}(s, t) .
$$

Among the three grayscale methods, $h(s, t)$ represents the grayscale value after $(s, t)$ conversion, and $z$ represents their respective weights.

Use the weighting method to grayscale the acquired image, and the corresponding relationship of the transformed matrix is as follows.

2.2.2. Image Denoising. Image smoothing is a commonly used method to improve image quality, such as spatial domain methods and frequency domain methods [25, 26].
Among them, the frequency domain filtering easily blocks the edge information of the image originally belonging to the high-frequency signal, resulting in the loss of the image edge. On the other hand, the time-consuming Fourier transform and Fourier transform filtering do not support real-time recognition [27, 28].

Mean filtering is given as follows.

Let $h(a, b)$ be a noisy image, and the denoised image $g(a, b)$ can be expressed as

$$
g(a, b)=\sum_{s=f}^{f} \sum_{t=f}^{f} h(a-s, b-t)
$$

where $f$ is the size of the image; then the total number of pixels in the image is

$$
g(a, b)=\frac{1}{n} \sum_{s=f}^{f} \sum_{t=f}^{f}(a+s, b+t), g(a, b)=\frac{1}{n} \sum_{s, t \in W} f(s, t),
$$

where $W$ represents the point set in the $(a, b)$ field.

The result of image filtering is mainly determined by the size of the template, so it is very important to choose a good template.
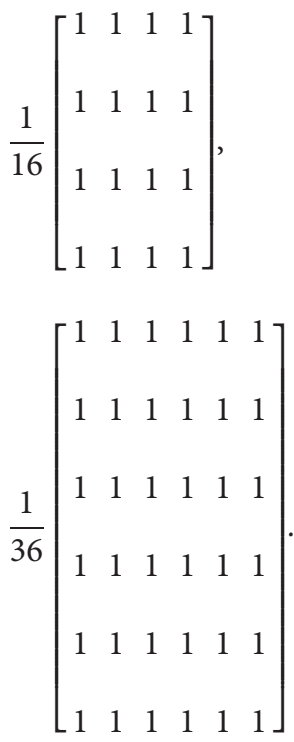

Machine vision technology allows certain camera models to transform the coordinate relationship between points in $3 \mathrm{D}$ space and points in $2 \mathrm{D}$ images. In practical applications, there are many camera models, which are generally divided into linear models and nonlinear models. The pinhole model is the most commonly used linear model. The expression formula of the pixel gray value of the image after filtering is

$$
k_{a}(a)=d^{-1} \iint g(a) c(b-a) s(g(a)-g(b)) .
$$

Here, $k$ and $a$, respectively, represent the gray value of the corresponding point after filtering and before filtering, and $g$ is a coefficient of determination, and its expression is 


$$
p(a)=\iint c(g-a) s(r(a)-g(a)) .
$$

$s$ represents the spatial similarity between the center point and the points in its neighborhood, which is defined as

$$
s(g-a)=e^{1 / 2(\|g-a\| / \phi)^{2}} .
$$

The results of edge information retention and noise reduction can be achieved by two factors that determine the coefficients of the filter method. One is the filter factor, which is directly related to the geometric spatial distance, and the other is the pixel. Double-sided filtering not only considers the relationship between the physical distances between pixels but also considers the similarity between the gray values of these pixels and each nearby point.

\section{Design Experiment of Civil Engineering Construction Safety Management System Based on Artificial Intelligence Machine Vision Technology}

\subsection{Safety Management Factors of Civil Engineering} Construction. On-site safety production process control, first of all, is the control of hazards and major hazards. It plays an active role in containing and reducing the occurrence of safety accidents, reducing safety risks, and promoting safety standards and excellence. However, work-related accidents often occur during the operation of construction workers, and work-related accidents are related to people. According to statistics, the safety management of workers in construction enterprises mainly has the following problems.

It is mainly manifested in the low safety quality of operators, backward concepts, inadequate safety production and operation techniques, outdated content and single form of safety education and training for construction personnel, and hidden dangers in safety supervision and management of construction personnel $[29,30]$. Because most of the construction workers are migrant workers without formal education, low education level, poor learning ability, low safety quality, and other factors, the construction personnel are not clear about the safety technical measures, safety operation specifications, and other protective knowledge on the site, leading to improper technical operations, forming potential safety hazards, and causing safety accidents for construction workers [31, 32]. In addition, safety education is not in place, and most of safety education and training ends in the form of a formality. The weakness of the basic work and the mere formality of safety activities have made the safety production rules and operating procedures not implemented in the front line of production. They are in vain and the effect of safety education is poor. The supervision in project management is not in place, and the potential safety hazards are not paid enough attention to, and there is a phenomenon of formalism, which leads to the occurrence of accidents. Among them, the types of civil engineering safety accidents are mainly collapses, falling from heights, object hits, mechanical injuries, and lifting injuries. Among them, collapse accidents are more frequent, accounting for a relatively large proportion. There are many safety factors, including human factors, mechanical factors, management factors, and environmental factors $[33,34]$.

\subsection{Artificial Intelligence Machine Vision Technology Recog-} nizes and Supervises Construction Safety. As an automated method, artificial intelligence machine vision technology depends on monitoring equipment to achieve automation. The machine replaces the human eye for measurement and judgment, converts the captured target into an image signal, and transmits it to a dedicated image processing system to obtain the captured image. The shape information of the target is converted into digital signals according to the pixel distribution, brightness, color, and other information; the image system performs various operations on these signals to extract the characteristics of the target and then controls the action of the equipment on the spot according to the results of the discrimination.

With the gradual development of machine vision technology and algorithmic classifiers, methods such as automatic camera recognition and monitoring worker behavior recognition have become increasingly mature. However, all vision-based technologies require a certain cost investment, such as relatively expensive cameras. At the same time, image processing technology is very sensitive to changes in light and background, and the complex scene environment adds considerable difficulty to the image information processing process. Please note that not all construction sites, such as some temporary buildings and ancillary facilities, are suitable for installing cameras, and it is difficult to ensure that the cameras cover all working surfaces, so this method is not common due to these factors. Therefore, in the daily construction process, not only must the camera be used to monitor the construction site, but also the risk monitoring implementers must regularly monitor various indicators based on the actual project, take necessary prevention and control measures, classify the types of risk objects, and implement risk monitoring. The personnel analyze the risk monitoring records and results, report the analyzed risks in time, and give early warning in time.

3.3. Construction Safety Management System. Take the largescale civil engineering construction safety management system as the detection object, and use the various subsystems, organizations, and modules in the safety management system as the detection elements to construct the detection index system of the large-scale civil engineering construction safety emergency management system, and establish the detection model. Implement inspections on the operation and maintenance of large-scale civil engineering construction safety management systems, investigate problems based on the inspection results, carry out defect diagnosis, explore dynamic emergency management detection mechanisms, constantly find hidden safety hazards, and make timely corrections to enhance the effectiveness of the emergency management system, reliability, and other functions. 
The use of mobile devices does not require additional costs, the built-in sensor is more stable than the image sensor, and the accuracy and power consumption are more reasonable. At the same time, smart terminal devices have powerful data storage, transmission, and processing capabilities, which bring significant advantages to job recognition automation. By monitoring the behavior of unsafe employees, accidents can be effectively prevented on-site. In order to solve the shortcomings of safety management behavior in traditional construction management, the construction of activity recognition and driving record system based on intelligent terminal is proposed. Construct a behavior recognition model, and use machine learning classification algorithms to intelligently recognize and record the behavior of workers on the construction site based on the traffic data automatically received by the smart terminal to achieve an increase in tasks.

This paper designs a civil engineering construction safety management system, which can monitor the three influencing factors of human, machine, and environment in the construction site, eliminate potential safety hazards of construction personnel, and avoid accidents. If there are potential safety hazards, the system will issue early warnings to construction personnel and management personnel in a timely manner. As shown in Figure 3, the system operation mechanism includes the three following stages:

(1) Model building stage: first, install the monitoring equipment in the center of the project, and collect the speed and angle information of the staff through highly integrated sensors, by collecting traffic samples of a large number of employees, creating behavioral perception or traffic recording models, and storing them on smart devices.

(2) Application implementation stage: the monitoring equipment calls the built-in identification unit of the equipment, analyzes the employee's action signal, and marks it as "safe" or "unsafe." Then, the employees and the management center will receive notifications or issue alarms based on the recognition results. If unsafe behavior occurs, the terminal will notify both. Supervise employees according to appropriate operating instructions, and the manager evaluates BBS risks based on on-site inspections and formulates weak link management strategies.

(3) Model update stage: by strengthening the application stage data, the standard error is input into the training sample database, and the classification model is updated and optimized in stages.

The main feature of this system is to transplant the concept of "crowdsourcing" to solve the sampling problem of traditional BBS. "Crowdsourcing" refers to publishing a certain task through the Internet, which is completed by the public, usually accompanied by a certain reward mechanism. Based on the online transmission function of the smart terminal (mobile, LAN, or Bluetooth), every construction worker with a smart terminal can provide training samples with tags, thereby establishing a large training sample database. Conversely, the construction personnel can download the model from the server to the terminal at any time to realize the model sharing. Through the use of artificial intelligence machine vision technology to monitor safety incidents, more effective and comprehensive safety management can be achieved. Make full use of intelligent terminals for data collection and real-time monitoring to realize low-cost automation. This method is highly scalable and can be extended to similar scenarios or fields, such as detecting or monitoring the construction status of personnel, structures, and machinery during the construction process.

3.4. Data Collection. There are no specific national operating standards or requirements, but, by consulting experienced site supervisors and seat belt manufacturers, at least one lifting point can prevent workers from high-altitude safety accidents when working at heights by understanding the need to connect to devices such as stable falls. In the vertical climbing process, employees choose to move the suspension points at intervals of 1-2 steps each time according to their comfort level and step height spacing during the climbing process. This collected the last 50 samples of climbing with a seat belt and 50 samples of climbing without a seat belt. Similarly, when moving horizontally on the scaffolding workbench, the safety belt attachment points alternately rotate along the scaffold grid, wearing 54 exercise samples and the safety belt of the subject wearing the safety belt.

3.5. Feature Extraction. In this study, we extracted 8 features, that is, a total of 57 attribute values, as shown in Table 1. Here, each characteristic of a signal is regarded as an "attribute," and attributes with the same definition belong to a kind of characteristic. For example, the maximum value of the acceleration signal on the $x$-axis is regarded as the attribute, and the maximum value of the acceleration or angle signal along different axes is considered to belong to the characteristic "maximum."

\section{Civil Engineering Safety Management Analysis Based on Artificial Intelligence Machine Vision Technology}

4.1. Best Feature Combination of Machine Vision. In order to reduce the dimension of the attribute table as much as possible to construct the best attribute table, reduce memory consumption, and ensure the best sorting performance, this experiment uses principal component analysis and highly correlated filters to compare and select them. Figure 4 shows the frequency distribution of the best attribute selected by the attribute selector.

In the Waikato environmental knowledge analysis workbench, high-correlation filtering is implemented through the attribute selector, and the predictive ability of each attribute and the degree of redundancy between them are evaluated separately, in order to select a group of highly correlated but low-correlation labels. In order to simplify the follow-up procedures, especially to transplant the analysis 


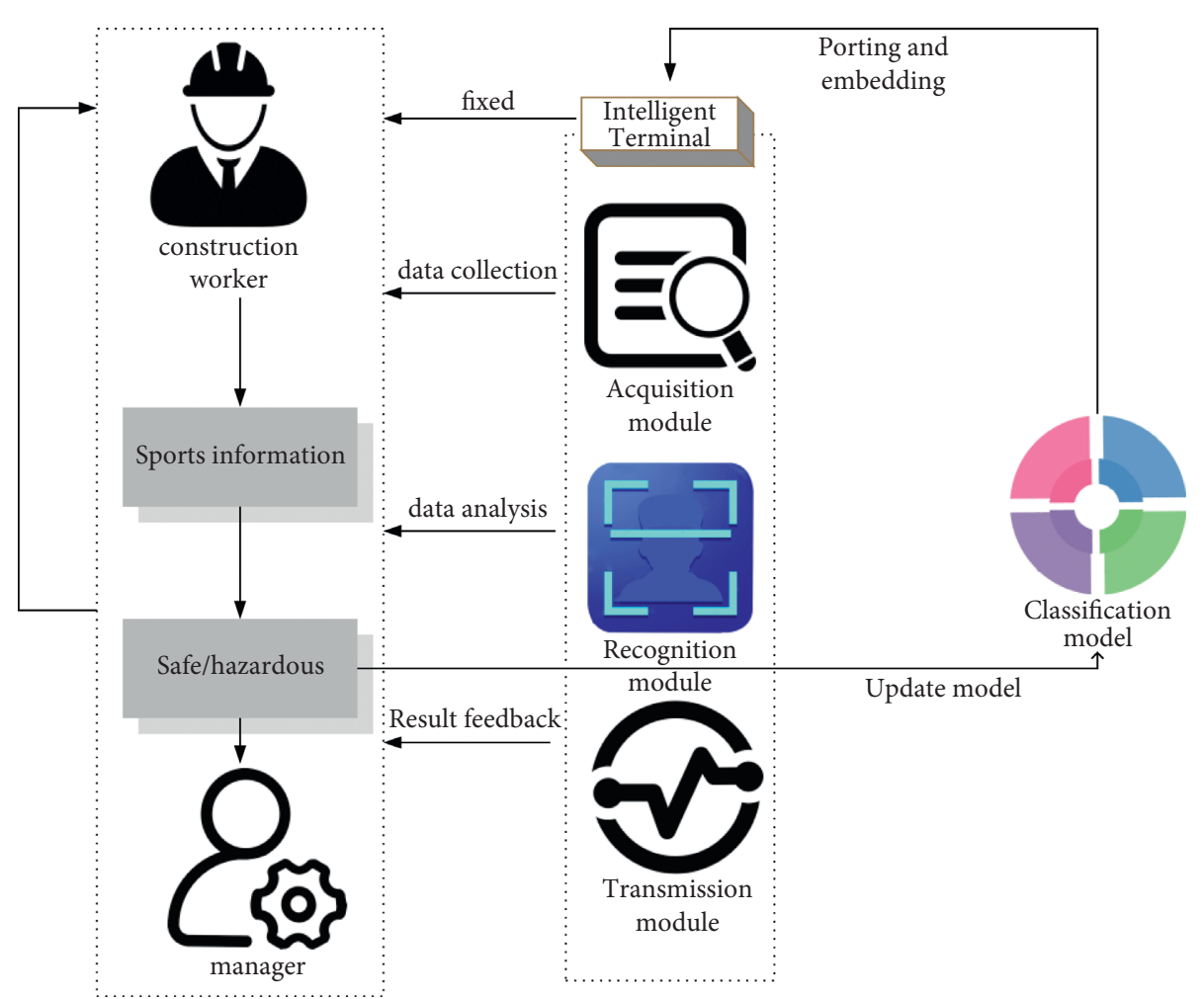

FIGURE 3: Civil engineering safety management intelligent identification system.

TABLE 1: Feature extraction type and serial number list.

\begin{tabular}{lcc}
\hline Number & Feature & Quantity \\
\hline 1 & Correlation coefficient & 6 \\
2 & Average power & 8 \\
3 & Signal entropy & 6 \\
4 & Peak frequency & 12 \\
5 & Absolute peak & 6 \\
6 & Absolute mean & 8 \\
7 & Form factor & 8 \\
8 & Square root amplitude & 3 \\
\hline
\end{tabular}

module to the smart mobile terminal, in this research, we established several feature combinations by comparing the frequencies of use of various attributes selected in the analysis results, which are called "best features" combinations. Table 2 shows the best combination of features.

In order to obtain the attenuation effect of the filtering algorithm in the image, $4 * 4$ and $6 * 6$ models were selected to reduce the noise of the noisy image, and the noise reduction time of each filter model was measured in turn. The result is shown in Figure 5.

From the subjective evaluation criteria, the image obtained by using the standard Gaussian filter is clearer and has less noise. From the various data in the figure, the time used for Gaussian filtering is relatively short, and the processing time is positively correlated with the size of the template. The lower the PSNR, the higher the distortion of the smoothed image and the greater the loss of image details. The performance comparison of detection is shown in Table 3.
4.2. Data Analysis. Figure 6 shows the acceleration and angle information of two different samples. The "positive" sample value is the exercise information of the experiment participants using the seat belt to climb the ladder vertically, while the "negative" sample represents the exercise information of not using the seat belt. From the time history curve, it is difficult to obtain rules or features to distinguish positive samples from negative samples. Therefore, it is still necessary to extract some signal characteristics to reveal the essential difference.

To evaluate different models, select 5 indicators, average 10 times the accuracy of cross-validation, and measure F, uptime, and memory consumption. AUC provides a trade-off between the percentage of true positives (the percentage of positive samples that are correctly identified) and the percentage of false positives (the percentage of negative samples that are incorrectly identified as positive samples). Similarly, the F1 value is to reconcile the average accuracy rate (i.e., the ratio of true positive samples to predicted positive samples) and the recall rate (i.e., the ratio of correctly identified positive samples to actual positive samples). The average accuracy rate refers to the average accuracy rate obtained from 10 mutual verifications, and the execution time is the total time required to use $2.7 \mathrm{GHz}$ Intel Core i5 CPU and $8 \mathrm{~GB}$ RAM. In theory, the higher the average accuracy is, the closer the AUC and F measured values are to 1.0, the shorter the running time is and the less memory consumption is, and the better the performance of the classification model is. 


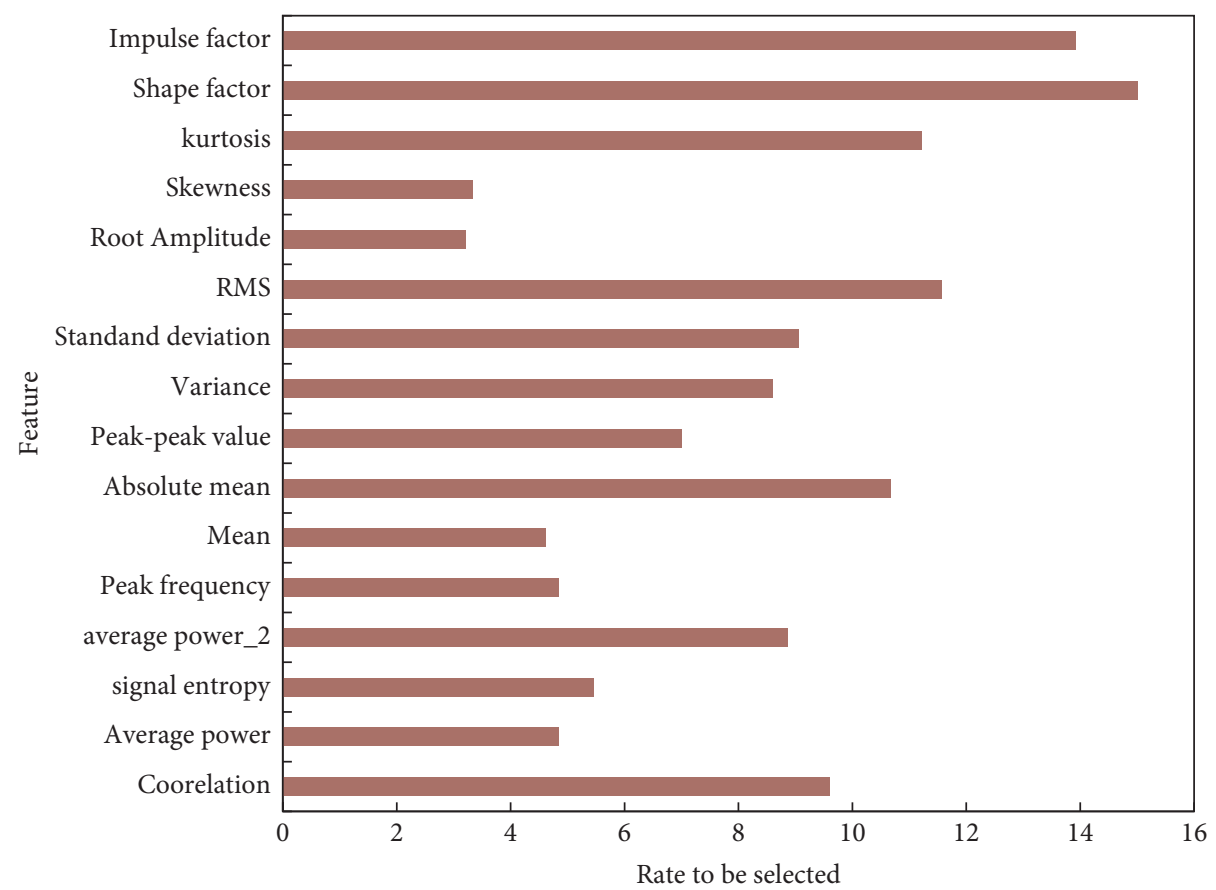

Figure 4: Frequency distribution of the best feature selected by the attribute selector.

TABle 2: Best feature combination.

\begin{tabular}{lcc}
\hline Combination name & Feature & Envelope attribute value number \\
\hline A1 & Correlation coefficient & 12 \\
B2 & Correlation coefficient, signal entropy & 24 \\
C3 & Correlation coefficient, signal entropy, standard deviation & 33 \\
D4 & Correlation coefficient, signal entropy, standard deviation, average power & 38 \\
\hline
\end{tabular}

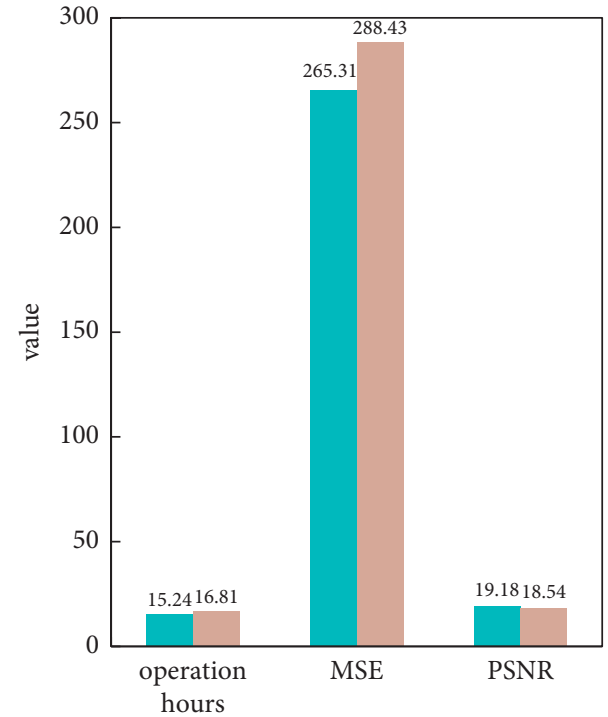

Factor

- $4^{\star} 4$

$6^{*} 6$

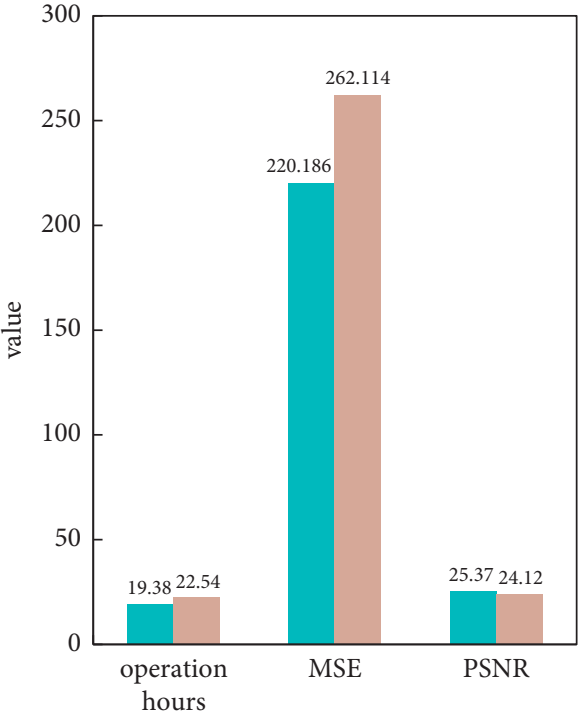

Factor

$4^{*} 4$

- $6^{*} 6$

Figure 5: Filtering effect comparison. 

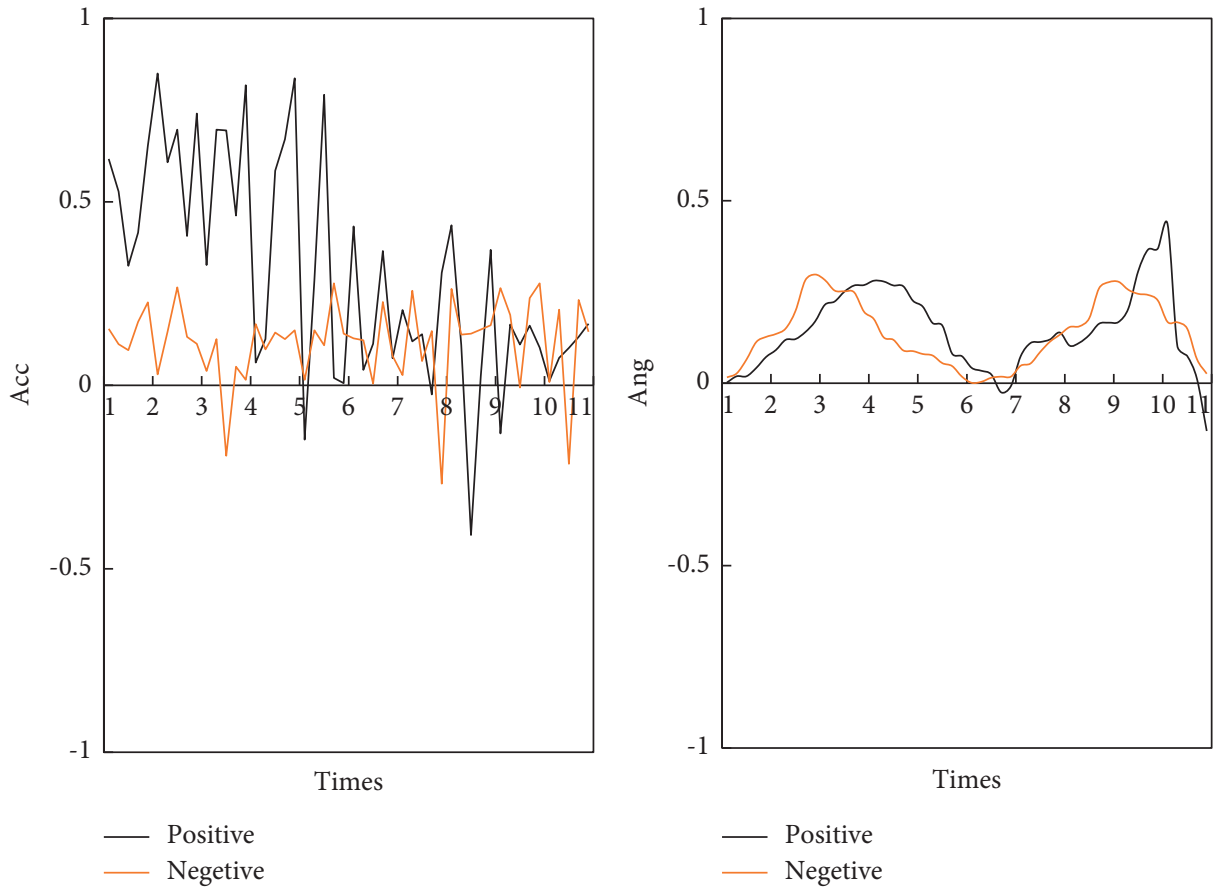

Figure 6: Acceleration and angle information.

TABle 3: Performance comparison of detection operators.

\begin{tabular}{lccc}
\hline Detection operator & Detection threshold & Actual inspection quantity & Running time (ms) \\
\hline SURF & 400 & 400 & 101.56 \\
SIFT & 400 & 400 & 1.32 \\
Fast & 400 & 400 & 559.47 \\
Shi-Tomasi & 400 & 400 & 380.13 \\
SURF & 800 & 400 & 103.55 \\
SIFT & 800 & 800 & 1.51 \\
Fast & 800 & 800 & 626.46 \\
Shi-Tomasi & 800 & 800 & 425.86 \\
\hline
\end{tabular}

4.3. Safety Management Factors in Civil Engineering Construction. The safety management of construction personnel is people-oriented. In order to ensure the safety of each of the construction personnel, it is necessary to consider the shortcomings and adopt advanced methods according to the actual situation of the construction site. The key factor affecting the safety management of construction personnel is whether the construction personnel have been scientifically managed, such as comprehensive, scientific, and effective supervision and management of construction personnel to eliminate hidden safety hazards for construction personnel.

In civil engineering, the four common factors are human, mechanical, environmental, and management factors. The survey format is used to survey people in the construction industry, investigate the reliability of the six major elements, and calculate the reliability. The results are shown in Table 4.
TABLE 4: Factor reliability survey.

\begin{tabular}{lc}
\hline Reliability & Cronbach alpha coefficient \\
\hline Human factor & 0.6152 \\
Mechanical factors & 0.7053 \\
Material factors & 0.7129 \\
Method factors & 0.7419 \\
Environmental factor & 0.6848 \\
Management factors & 0.6536 \\
Overall reliability & 0.6877 \\
\hline
\end{tabular}

Analyze from the four perspectives of human, machine, environment, and management, and summarize the subfactors of each of the four major factors. The results are shown in Figures 7 and 8.

Combined with the characteristics of construction personnel safety management, 16 individual factors among the four key factors of human, mechanical, environment, and management are quantified. The workload is very large. From the single factors among the four 

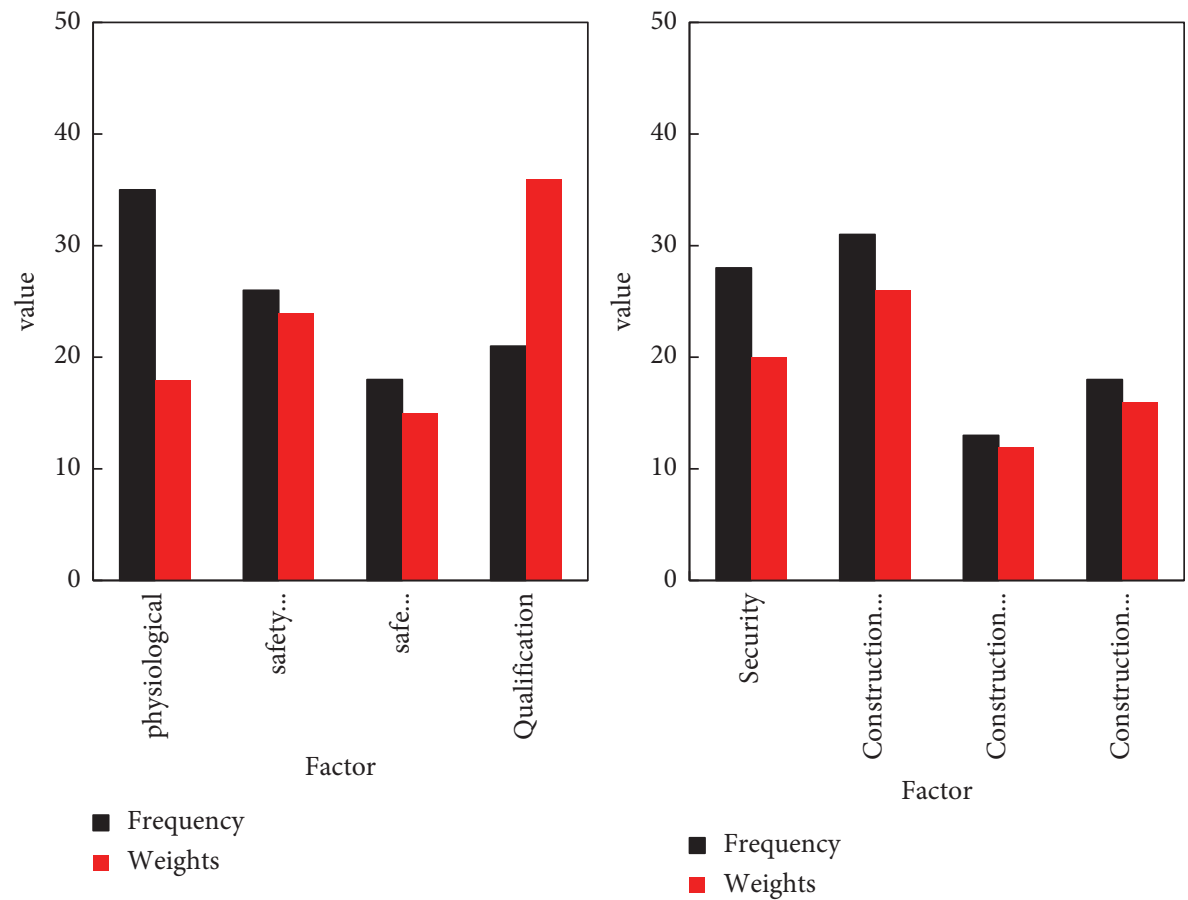

FIgURE 7: Frequency statistics of human and machine factors.
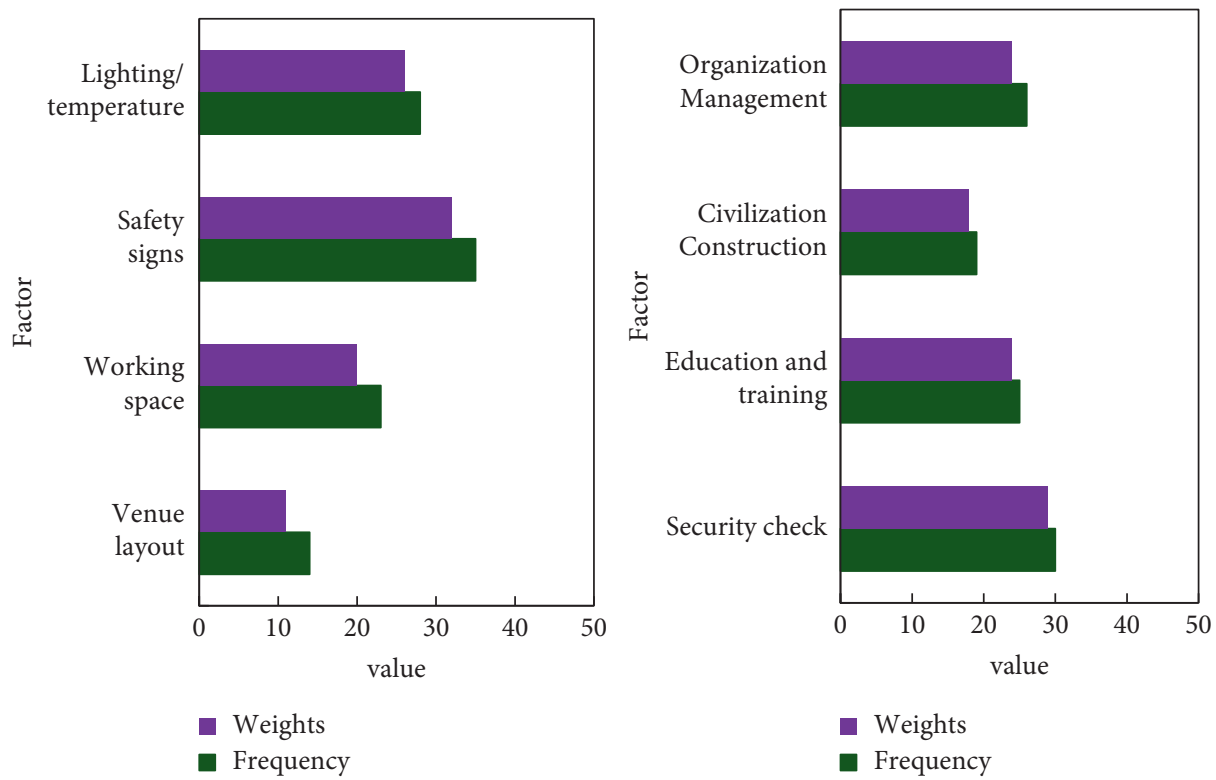

Figure 8: Frequency statistics of environmental management factors.

TABLE 5: Single factor index data quantification processing results. 


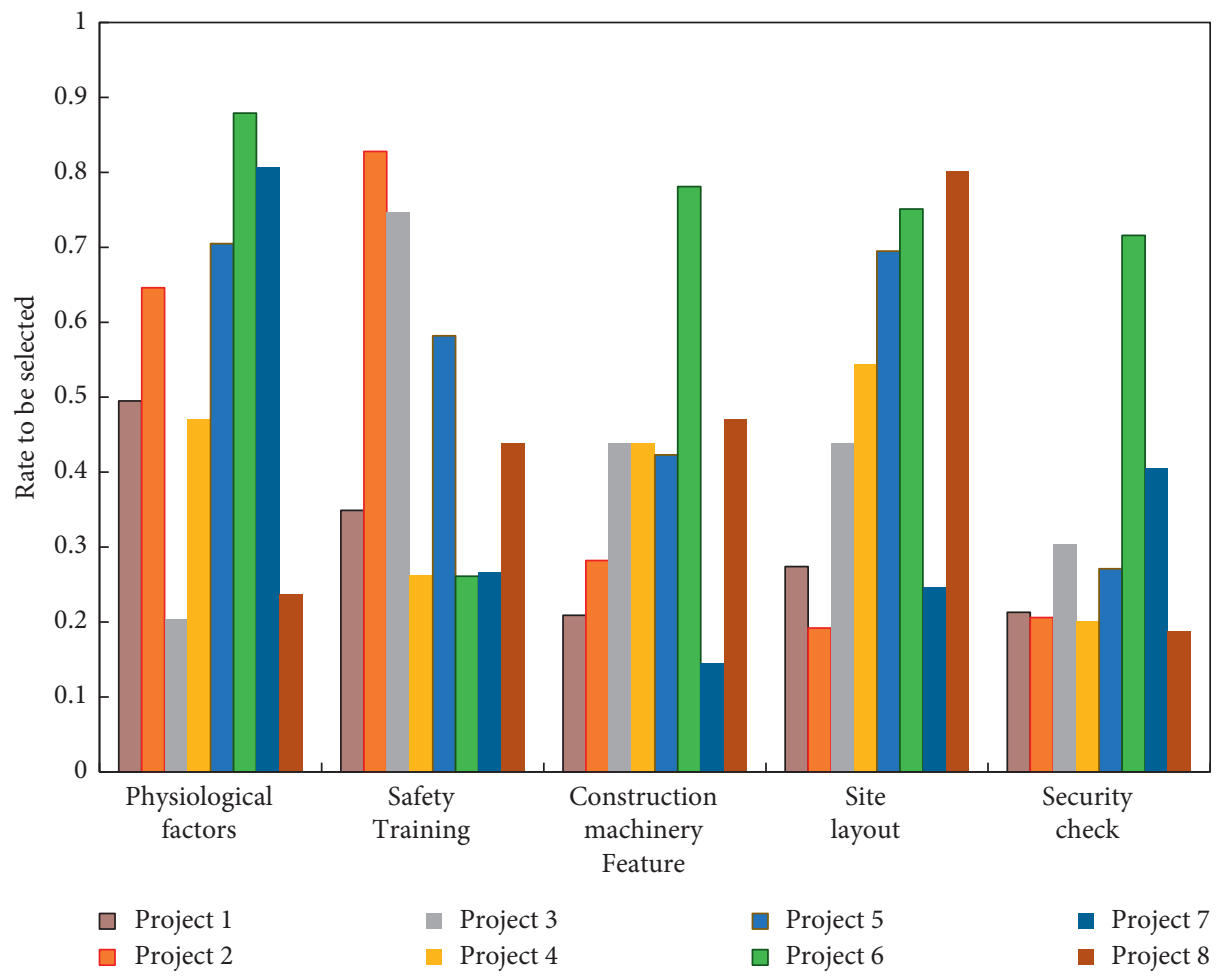

FIGURE 9: Sample data normalization processing result.

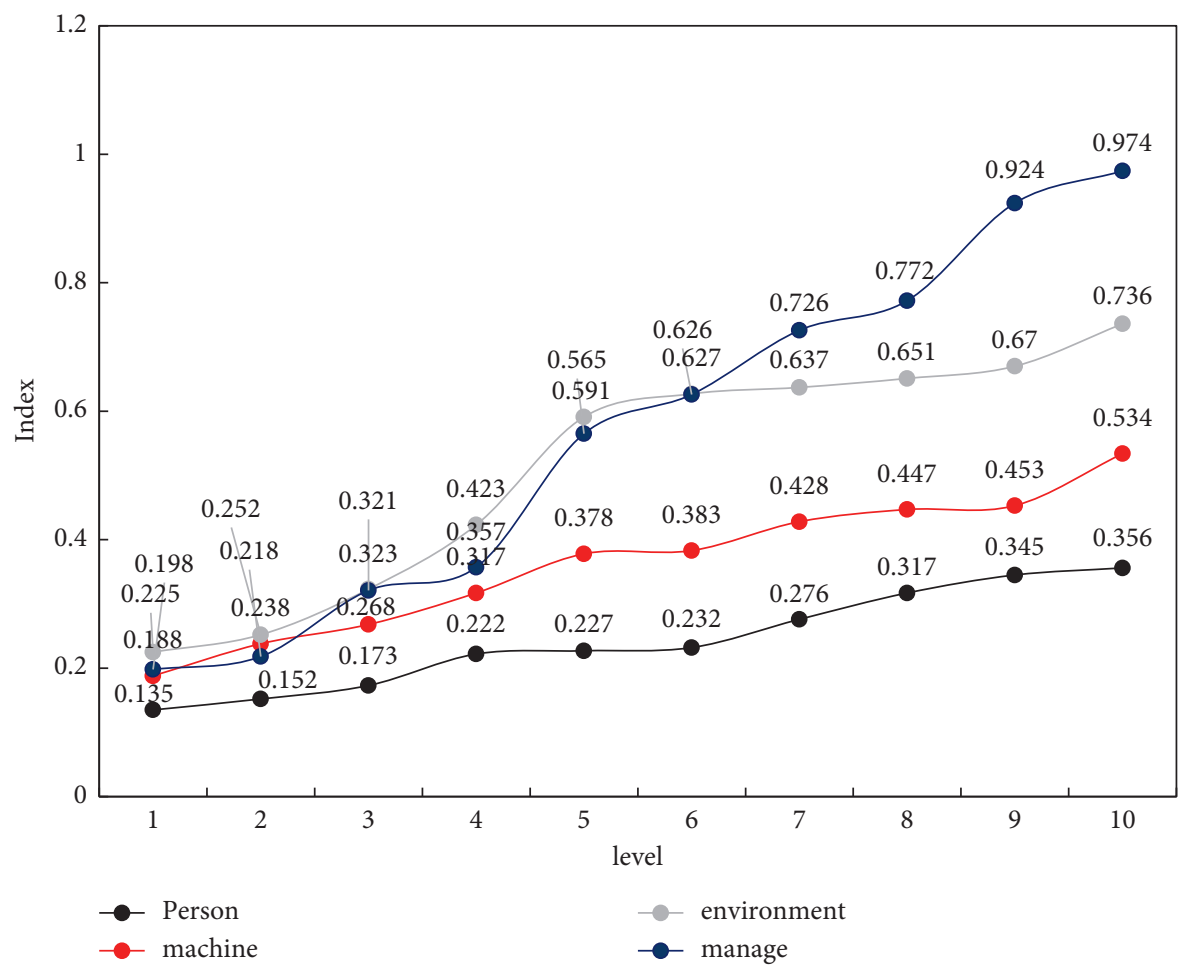

FIgURE 10: Comprehensive level of civil engineering construction safety management.

key factors, select one of the single factors with the greatest impact. Human factors are the most important factors in quantification. Therefore, two separate factors are used to represent quantified human safety factors.
That is, five independent factor indicators are selected as control points: physiological factors, training, and safety training, as shown in Table 5 for site layout and safety inspection. 
Since the collected data must have the characteristics of authenticity and easy access, the five selected indicators must be quantified, using a percentile system to quantify the status, and judge whether the status is good.

\subsection{Effectiveness of Civil Engineering Construction Safety} Management. Through the investigation on the site of other construction projects under construction, the relevant data of the safety management of these 6 construction sites as samples are summarized, as shown in Figure 9.

At the construction site, among the four key factors affecting the safety management of construction personnel, human factors are often the key factors affecting the entire system. Depending on the three elements of management agent, man-machine, and environment, security is directly related to management. Therefore, the higher the score of the target layer in the building, the greater the impact on the safety management of civil engineering construction personnel. Figure 10 shows the complete civil engineering construction safety management level based on artificial intelligence machine vision technology.

Increasing the influence of human factors and management factors will rapidly improve the overall level of safety management, and increasing the influence of management factors will improve the level of integration of safety management, followed by an increase in human factors. The impact of environmental conditions, materials, and machinery is unclear. Other safety management factors have not changed, but increasing the safety investment of one of the safety factors will correspondingly improve the safety management level of the civil engineering site, increasing the safety management level to $97.4 \%$ and reducing the time required to reach the target value of the safety control level.

\section{Conclusions}

This paper takes safety management as the main line and systematically studies the whole process of emergency management such as prevention, preparation, response, and recovery of civil engineering construction safety incidents, aiming to study the ideas and methods of largescale civil engineering construction safety management, and improves the construction safety incident management theory, promote the construction of emergency response capacity from a practical level, and improves the level of safety management. This article is based on artificial intelligence machine vision technology to study the application of civil engineering construction safety management, using artificial intelligence machine vision technology to monitor the construction of workers to ensure the safety of civil engineering construction. In the research of this article, the safety management level of civil engineering structure has been successfully improved. Improving the safety level of civil engineering structures is a long-term and time-consuming process. The improvement of safety awareness and operational skill proficiency required for achieving the desired goal of the safety management level has a process. There are still many shortcomings in this research work, such as interference with the artificial intelligence machine vision technology designed in this paper at the construction site, insufficient light, and other complex conditions that lead to recognition errors. The monitoring may also be unstable due to the factors of the construction environment. The civil engineering construction safety management studied in this article can meet the general construction environment, but, for the harsher environment, further research is needed. This article only focuses on human factors, which are the main factors that affect the safety management of construction workers, and there are relatively few studies on other factors that affect the safety of construction workers. When tracking a trajectory, there is at most one person and one object. If multiple similar targets are displayed at the same time, other target control methods should be used for trajectory tracking to ensure target accuracy and avoid position coordinates. Moreover, under different environmental factors and weather factors, the recognition may be inaccurate and the characteristic information cannot be extracted correctly. Moreover, we will further study how to establish the quantitative relationship between the coordinates and distances of image pixels and the actual coordinates and distances to achieve the purpose of reflecting the actual distances and trajectories between targets.

\section{Data Availability}

The data that support the findings of this study are available upon request to the author.

\section{Conflicts of Interest}

The author declares no conflicts of interest with respect to the research, authorship, and/or publication of this article.

\section{References}

[1] A. Zl, A. Dc, A. Rl, and B. Aa, "Artificial intelligence for securing industrial-based cyber-physical systems," Future Generation Computer Systems, vol. 117, pp. 291-298, 2021.

[2] H Wei and N Kehtarnavaz, "Semi-supervised faster rcnnbased person detection and load classification for far field video surveillance," Machine Learning and Knowledge Extraction, vol. 1, no. 3, pp. 756-767, 2019.

[3] X. W. Ye, C. Z. Dong, and T. Liu, "A review of machine visionbased structural health monitoring: methodologies and applications," Journal of Sensors, vol. 2016, no. 5, 10 pages, Article ID 7103039, 2016.

[4] S. Cubero, W. S. Lee, N. Aleixos, F. Albert, and J. Blasco, "Automated systems based on machine vision for inspecting citrus fruits from the field to postharvest-a review," Food and Bioprocess Technology, vol. 9, no. 10, pp. 1623-1639, 2016.

[5] A. A. Robie, K. M. Seagraves, S. E. R. Egnor, and K. Branson, "Machine vision methods for analyzing social interactions," Journal of Experimental Biology, vol. 220, no. 1, pp. 25-34, 2017.

[6] D Benny and D Jaishree, "Construction safety management and accident control measures," International Journal of Civil Engineering \& Technology, vol. 8, no. 4, pp. 611-617, 2017. 
[7] D Nkurunziza, "Investigation into road construction safety management techniques," Open Journal of Safety Science and Technology, vol. 10, no. 3, pp. 81-90, 2020.

[8] W. Zhang, X. Zhang, X. Luo, and T. Zhao, "Reliability model and critical factors identification of construction safety management based on system thinking," Journal of Civil Engineering and Management, vol. 25, no. 4, pp. 362-379, 2019.

[9] L. Qiao, Y. Li, D. Chen, S. Serikawa, M. Guizani, and Z. Lv, “A survey on 5G/6G, AI, and Robotics," Computers \& Electrical Engineering, vol. 95, Article ID 107372, 2021.

[10] M. A. Rebelo, F. R. Silveira, E. Czarnocka, and K. Czarnocki, "Construction safety on scaffolding: building information modeling (BIM) and safety management," U Porto Journal of Engineering, vol. 5, no. 2, pp. 46-60, 2019.

[11] W. S Qureshi, A Payne, K. B Walsh, R Linker, O Cohen, and M. N Dailey, "Machine vision for counting fruit on mango tree canopies," Precision Agriculture, vol. 17, no. 3, pp. 1-21, 2016.

[12] P. F. Shan*, "Image segmentation method based on K-mean algorithm," EURASIP Journal on Image and Video Processing, vol. 2018, no. 1, p. 81, 2018.

[13] T.-H. Sun, F.-C. Tien, F.-C. Tien, and R.-J. Kuo, "Automated thermal fuse inspection using machine vision and artificial neural networks," Journal of Intelligent Manufacturing, vol. 27, no. 3, pp. 639-651, 2016.

[14] S. Ghosal, D. Blystone, A. K. Singh, B. Ganapathysubramanian, A. Singh, and S. Sarkar, "An explainable deep machine vision framework for plant stress phenotyping," Proceedings of the National Academy of Sciences, vol. 115, no. 18, pp. 4613-4618, 2018.

[15] B. Wang, B. F. Zhang, X. W. Liu, and F. C. Zou, "Novel infrared image enhancement optimization algorithm combined with DFOCS," Optik, vol. 224, Article ID 165476, 2020.

[16] C. S Nandi, B Tudu, and C Koley, "A machine vision technique for grading of harvested mangoes based on maturity and quality," IEEE Sensors Journal, vol. 16, no. 16, p. 1, 2016.

[17] L. Y Chang, H. E San-Peng, Q Liu, J Xiang, and H Danfeng, "Quantifying muskmelon fruit attributes with A-TEP-based model and machine vision measurement," Journal of Integrative Agriculture, vol. 17, no. 6, pp. 1369-1379, 2018.

[18] R. M. Nordin, N. A. Jasni, N. A. Abdul Aziz, N. Hashim, Z. Ismail, and J. Yunus, "Construction safety management system at project level using system dynamic model (SDM)," Engineering Journal, vol. 25, no. 1, pp. 221-232, 2021.

[19] S. Jeschke, C. Brecher, H. Song, and D. Rawat, Industrial Internet of Things: Cybermanufacturing Systems, pp. 1-715, Springer, Switzerland, Europe, 2017.

[20] Q Qihu and L Peng, "Safety risk management of underground engineering in China: progress, challenges and strategies ScienceDirect," Journal of Rock Mechanics and Geotechnical Engineering, vol. 8, no. 4, pp. 423-442, 2016.

[21] C.-L. Chung, K.-J. Huang, S.-Y. Chen, M.-H. Lai, Y.-C. Chen, and Y.-F. Kuo, "Detecting Bakanae disease in rice seedlings by machine vision," Computers and Electronics in Agriculture, vol. 121, pp. 404-411, 2016.

[22] T. Dawood, Z. Zhu, and T. Zayed, "Machine vision-based model for spalling detection and quantification in subway networks," Automation in Construction, vol. 81, pp. 149-160, 2017.

[23] R. Rajashekar and B. M. Rajaprakash, "Development of a model for friction stir weld quality assessment using machine vision and acoustic emission techniques," Journal of Materials Processing Technology, vol. 229, pp. 265-274, 2016.
[24] B. Wang, B. F. Zhang, and X. W. Liu, "An image encryption approach on the basis of a time delay chaotic system," Optik International Journal for Light and Electron Optics, vol. 225, Article ID 165737, 2021.

[25] A. Yang, X. Gao, and M. Li, "Design of apochromatic lens with large field and high definition for machine vision," Applied Optics, vol. 55, no. 22, pp. 5977-5985, 2016.

[26] W. Tang, L. Tian, and X. Zhao, "Research on displacement measurement of disk vibration based on machine vision technique," Optik, vol. 127, no. 8, pp. 4173-4177, 2016.

[27] A. Suraj, K. Manoj, Q. Zhang, and D. W. Matthew, "Automated detection of branch shaking locations for robotic cherry harvesting using machine vision," Robotics, vol. 6, no. 4, p. 31, 2017.

[28] Q. Wang, B. Chen, and D. Zhu, "Machine vision-based selection machine of corn seed used for directional seeding," Nongye Jixie Xuebao/Transactions of the Chinese Society of Agricultural Machinery, vol. 48, no. 2, pp. 27-37, 2017.

[29] D.-m. Tsai and Y.-c. Hsieh, "Machine vision-based positioning and inspection using expectation-maximization technique," IEEE Transactions on Instrumentation and Measurement, vol. 66, no. 11, pp. 2858-2868, 2017.

[30] V Chauhan and B Surgenor, "Fault detection and classification in automated assembly machines using machine vision," International Journal of Advanced Manufacturing Technology, vol. 90, no. 9-12, pp. 2491-2512, 2017.

[31] F. You, Y.-h. Li, L. Huang, K. Chen, R.-h. Zhang, and J.-m. Xu, "Monitoring drivers' sleepy status at night based on machine vision," Multimedia Tools and Applications, vol. 76, no. 13, Article ID 14869, 2017.

[32] N. A Siddiqui, S. M Tauseef, and S. A Abbasi, "Advances in fire and process safety," Springer Transactions in Civil and Environmental Engineering, pp. 171-181, 2018.

[33] Y. Li, Y. Zuo, H. Song, and Z. Lv, "Deep learning in security of internet of things," IEEE Internet of Things Journal, vol. 2021, no. 99, p. 1, 2021.

[34] Z. Lv and H. Song, "Trust mechanism of feedback trust weight in multimedia network," ACM transactions on multimedia computing, communications, and applications, vol. 17, 2021. 